Modeling, Identification and Control, Vol. 42, No. 2, 2021, pp. 83-99, ISSN 1890-1328

\title{
Residual vibration control for robotic 3D scanning with application to inspection of marine propellers
}

\author{
E.B. Njaastad ${ }^{1}$ G.O. Tysse ${ }^{2}$ O. Egeland ${ }^{1}$ \\ ${ }^{1}$ Department of Mechanical and Industrial Engineering, Norwegian University of Science and Technology, N-7491 \\ Trondheim, Norway. E-mail: \{eirik.njaastad,olav.egeland\}@ntnu.no \\ ${ }^{2}$ Department of Production Technology, SINTEF Manufacturing AS, N-7465 Trondheim, Norway. E-mail: \\ geir.ole.tysse@sintef.no
}

\begin{abstract}
This paper presents a system for 3D scanning of a large marine propeller blade with a 3D camera mounted on an industrial robot. An industrial 3D camera with structured light is used where the accuracy is in the order of $0.1 \mathrm{~mm}$. The camera is mounted on a rod attached to the robot's end-effector to have sufficient reach for scanning the propeller. This rod introduces mechanical vibrations in the system when the robot is repositioned for a new scan. Fast and efficient scanning is achieved by using vibration cancellation in a feedforward configuration based on input shaping, where the programmed pose increments of the robot are reshaped to give a fast vibration settling time after repositioning the camera. The use of input shaping techniques ensures that the imaging device is at rest during the scanning operation when the object's surface is captured. Three different input shapers are considered: The Zero Vibration (ZV), ZV Derivative (ZVD), and Extra Insensitive (EI) shapers. By minimizing the residual vibrations, the accuracy and precision of the system are increased, and complete 3D scanning of objects can be performed in a shorter time. Moreover, the resulting scan quality is improved. The effectiveness of the proposed method is validated in simulations and experiments where the ZVD and EI shapers proved to be best suited for the scanning application. The experimental validation involved a full scanning operation for a marine propeller blade where a UR10 robot with the original industrial control system was used. It was seen that the proposed system gave sufficient accuracy for determining the surface of the propeller blade.
\end{abstract}

Keywords: Input shaping, Robotic 3D scanning

\section{Introduction}

Acquisition of accurate three-dimensional (3D) data is of high importance in manufacturing operations like design, machining, inspection, navigation, and control. 3D sensors are increasingly used for surface scanning due to the availability of commercial sensors with high accuracy. The benefit of a 3D camera compared to a 2D camera is that depth information is obtained, which gives additional information on the dimensions and pose of objects in a scene. Due to the limited view of the $3 \mathrm{D}$ camera, it may be necessary to fix the $3 \mathrm{D}$ camera to a robot, then move it to a sequence of different poses so that the data from the $3 \mathrm{D}$ camera can be used for a full reconstruction of the object of interest. The 3D camera must be repositioned and reoriented by the robot for each configuration in the scanning view sequence. A planning system is needed to generate a set of views that will give a sufficient characterization of the object's surface. The planning may result in a large number of scanning poses, which may cause the scanning operation to be time-consuming. In particular, it is a problem that the scanning must typically be done with a stationary camera, with the robot at rest 
during the scanning. An overview of this topic can be found in the review papers of Chen et al. (2000); Savio et al. (2007); Pérez et al. (2016); Schmitt et al. (2016).

In the scanning of large objects like a ship propeller, the limited motion range of an industrial robot may be a restricting factor. It is thus attractive to attach the camera to an extension of the arm, which will increase the coverage of the $3 \mathrm{D}$ scanning operation. The potential problem with this type of arrangement is that an extension of the arm will introduce additional elasticity to the robot arm, and there may be a significant settling time for the mechanical vibrations that are introduced in this way. This effect is critical for the scanning operation since the camera has to be at rest during the scanning. In an industrial setting, with a considerable amount of camera scanning poses, the accumulated settling time may violate the production cycle time requirements.

The mechanical vibrations introduced by the mechanical extension of the arm may be handled with active vibration damping using feedback control, which is a well-established technique. It is well known that if collocated sensors and actuators are used, then a simple passive feedback controller gives robust and efficient vibration damping (Joshi, 1989; Kanestrom and Egeland, 1994; Preumont, 2011). In the application studied in this paper, an industrial robot will be used with the original robot control system. Then the actuators will be at the robot joints, and collocation cannot be ensured. Moreover, the input to the industrial robot control system is not necessarily suited for active vibration control.

In this application, the mechanical vibrations are mainly caused by the motion between the scanning positions. This makes it interesting to investigate input shaping methods, where the trajectory input to the robot is modified to reduce the resulting vibrations. Input shaping is a feedforward method, which means that there are no stability problems, but a sufficiently accurate model of the dynamics of the elastic vibration must be available. Input shaping methods have been developed in great detail by Singer and Seering (1990); Kamel et al. (2008); Yu Zhao et al. (2016), and these shapers have been used for vibration damping in robots, cranes, space manipulators, and space antennas. Input shaping has also been successfully applied in inspection tasks for reducing residual vibrations in high-speed white light interferometry (Mun et al., 2015; Song et al., 2018) and atomic force microscopy (Schitter et al., 2008).

In this paper, we will use existing input shapers. The contribution of the paper is to investigate the efficiency of input shapers for a robotic scanning application, where the acquisition and reconstruction of the geome- try of marine propellers are achieved. Also, it is investigated how this can be implemented for an industrial robot with a commercial control system. Moreover, it is investigated if an industrial $3 \mathrm{D}$ camera on a robot can be used to scan a large marine propeller with sufficient accuracy.

The rest of this paper is organized as follows. Section 2 presents the geometry of robotic 3D scanning using structured light. In Section 3, a presentation of input shapers is given. The implementation and use of input shapers for a robotic measuring system are discussed in Section 4, while an experimental validation is presented in Section 5 before the conclusion in Section 6 .

\section{Geometry of robotic 3D scanning}

\subsection{Introduction}

This section presents the geometric background for surface determination by a 3D camera on a robot. In order to scan a large object like a ship propeller, the scanning data must be referenced to the same world frame. This requires hand-eye calibration, where the relative pose of the camera with respect to the robot end-effector is determined. This relative pose must be known and constant during scanning operations. However, these constraints might be violated in the presence of vibrations. The geometry of 3D scanning with structured light is similar to the geometry of stereo vision, and this is explained in the following to make it easier to understand the requirements of 3D scanning for surface determination.

\subsection{Hand-eye calibration}

A camera mounted on a robot can be used to measure the surface geometry of a target in camera coordinates. The motion commands of the robot are expressed in the coordinate frame of the robot. Therefore, extrinsic calibration between the optical frame of the camera and a robot coordinate system is critical for aligning the surface scans. Finding the transformation between the end-effector frame and the camera frame for a manipulator is considered as a hand-eye calibration. The problem was first formulated by Shiu and Ahmad (1989) and Tsai and Lenz (1989). The hand-eye transformation is needed in many sensing-acting tasks for robots, where we explicitly want to know the transformation $\boldsymbol{T}_{c}^{e}$ of the camera frame $c$ relative to the end-effector frame $e$.

Let $\boldsymbol{T}_{e}^{0}$ be the homogeneous transformation matrix from the base frame 0 to the end-effector frame $e$, which is a function of the vector $\boldsymbol{q}$ of joint variables, and let 


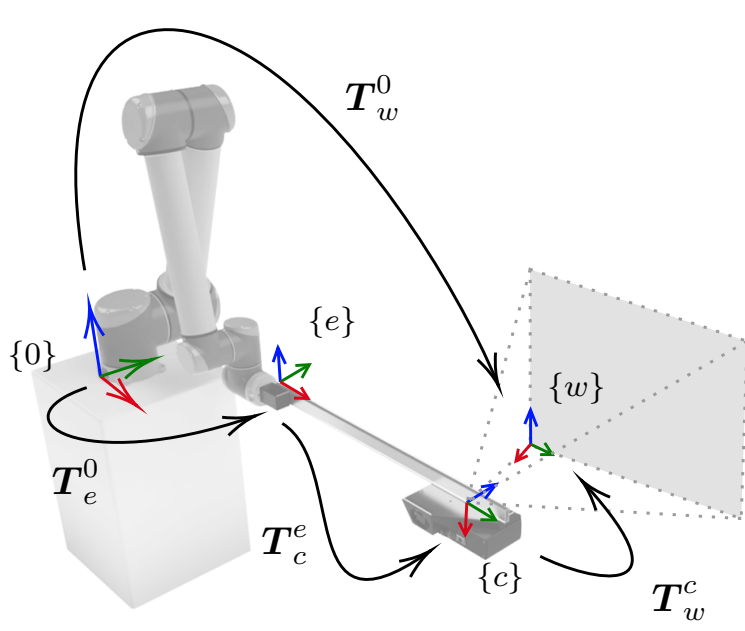

Figure 1: Coordinate transformations of a robotic 3D scanning system.

$\boldsymbol{T}_{w}^{c}$ be the transformation from the camera frame $c$ to a target frame $w$. Then, the position and orientation of a target frame $w$ with respect to the base frame 0 is given by $\boldsymbol{T}_{w}^{0}=\boldsymbol{T}_{e}^{0} \boldsymbol{T}_{c}^{e} \boldsymbol{T}_{w}^{c}$. This is illustrated in Figure 1 .

Consider the case where the robot is moved between a pair of configurations $\boldsymbol{q}_{i}$ and $\boldsymbol{q}_{j}$ with resulting homogeneous transformation matrices $\boldsymbol{T}_{e_{i}}^{0}$ and $\boldsymbol{T}_{e_{j}}^{0}$ for the robot, and $\boldsymbol{T}_{w}^{c_{i}}$ and $\boldsymbol{T}_{w}^{c_{j}}$ for the vision system. Since the frame $w$ is not moved, and $\boldsymbol{X}=\boldsymbol{T}_{c}^{e}$ is the same for both configurations, it follows that

$$
\boldsymbol{T}_{w}^{0}=\boldsymbol{T}_{e_{i}}^{0} \boldsymbol{X} \boldsymbol{T}_{w}^{c_{i}}=\boldsymbol{T}_{e_{j}}^{0} \boldsymbol{X} \boldsymbol{T}_{w}^{c_{j}} .
$$

Then an equation for the unknown transformation is found by defining

$$
\begin{aligned}
& \boldsymbol{A}=\left(\boldsymbol{T}_{e_{j}}^{0}\right)^{-1} \boldsymbol{T}_{e_{i}}^{0}=\boldsymbol{T}_{e_{i}}^{e_{j}} \\
& \boldsymbol{B}=\boldsymbol{T}_{w}^{c_{j}}\left(\boldsymbol{T}_{w}^{c_{i}}\right)^{-1}=\boldsymbol{T}_{c_{i}}^{c_{j}}
\end{aligned}
$$

which gives

$$
\boldsymbol{A X}=\boldsymbol{X} \boldsymbol{B}
$$

When $n \geq 2$ pairs of configurations are used, there will be one equation $\boldsymbol{A}_{k} \boldsymbol{X}=\boldsymbol{X} \boldsymbol{B}_{k}$ for each pair $k=$ $1, \ldots, n$, and the solution for $\boldsymbol{X}$ is found from

$$
\begin{gathered}
\boldsymbol{A}_{1} \boldsymbol{X}=\boldsymbol{X} \boldsymbol{B}_{1} \\
\vdots \\
\boldsymbol{A}_{n} \boldsymbol{X}=\boldsymbol{X} \boldsymbol{B}_{n}
\end{gathered}
$$

which can be solved as described in Park and Martin (1994). The displacement between the two configurations of pair $k$ as given by $\boldsymbol{A}_{k}$ and $\boldsymbol{B}_{k}$ can be described as a rotation $\theta_{k}$ about line $L_{k}$ and a translation $d_{k}$ along the same line (Daniilidis, 1999). The pairs of configurations should be selected so that the angles $\theta_{k}$ are large, and the pairs should be selected so that the angles between the lines $L_{k}$ are large (Tsai and Lenz, 1989). The matrix $\boldsymbol{A}_{k}=\boldsymbol{T}_{e_{i}}^{e_{j}}$ can be found from the forward kinematics of the manipulator in the two configurations $\boldsymbol{q}_{i}$ and $\boldsymbol{q}_{j}$.

The matrix $\boldsymbol{B}_{k}=\boldsymbol{T}_{c_{i}}^{c_{j}}$ can be found with a $2 \mathrm{D}$ camera using pose estimation techniques where the pose $\boldsymbol{T}_{w}^{c_{i}}$ and $\boldsymbol{T}_{w}^{c_{j}}$ of a calibration rig is determined (Tsai and Lenz, 1989). In this work we use the method of Umeyama (1991) to estimate $\boldsymbol{B}_{k}$ where $\boldsymbol{T}_{c_{i}}^{c_{j}}$ is determined directly with a $3 \mathrm{D}$ camera. The $3 \mathrm{D}$ camera gives us direct measurements of points on the surface of the target object. The displacement $\boldsymbol{T}_{c_{i}}^{c_{j}}$ between the camera frames $c_{i}$ and $c_{j}$ can be calculated by measuring the position of $N$ points fixed on the target object with respect to the reference frame $c_{i}$, and comparing them with the position of the corresponding $N$ points found on the same object with respect to the other camera frame $c_{j}$. Then the displacement

$$
\boldsymbol{T}_{c_{i}}^{c_{j}}=\boldsymbol{T}=\left[\begin{array}{cc}
\boldsymbol{R} & \boldsymbol{t} \\
\mathbf{0}^{T} & 1
\end{array}\right]
$$

from $c_{j}$ to $c_{i}$ can be determined from a sufficient number of points in general position.

Let $\boldsymbol{x}_{l}$ denote the position of the point $l$ in camera frame $c_{i}$, and let the same point hold position

$$
\boldsymbol{y}_{l}=\boldsymbol{R} \boldsymbol{x}_{l}+\boldsymbol{t}
$$

in reference frame $c_{j}$. When the point locations $\boldsymbol{x}_{j}$ and $\boldsymbol{y}_{l}$ are given for $l=1, \ldots, N$, the problem becomes finding $\boldsymbol{T}$, which can be done by using the cost function

$$
F_{1}=\sum_{l=1}^{N}\left\|\boldsymbol{y}_{l}-\left(\boldsymbol{R} \boldsymbol{x}_{l}+\boldsymbol{t}\right)\right\|^{2} .
$$

The minimization is done in two steps. First $\boldsymbol{R}$ is found, and then $\boldsymbol{t}$ is found for this value of $\boldsymbol{R}$. The problem is simplified by writing

$$
\boldsymbol{y}_{j}=\overline{\boldsymbol{y}}+\delta \boldsymbol{y}_{j}, \quad \boldsymbol{x}_{j}=\overline{\boldsymbol{x}}+\delta \boldsymbol{x}_{j}
$$

where

$$
\overline{\boldsymbol{y}}=\frac{1}{N} \sum_{j=1}^{N} \boldsymbol{y}_{j}, \quad \overline{\boldsymbol{x}}=\frac{1}{N} \sum_{j=1}^{N} \boldsymbol{x}_{j}
$$

are the centroids of the two point sets. It follows that $\overline{\boldsymbol{y}}=\boldsymbol{R} \overline{\boldsymbol{x}}_{j}+\boldsymbol{t}$, which gives

$$
\delta \boldsymbol{y}_{j}=\boldsymbol{R} \delta \boldsymbol{x}_{j}
$$




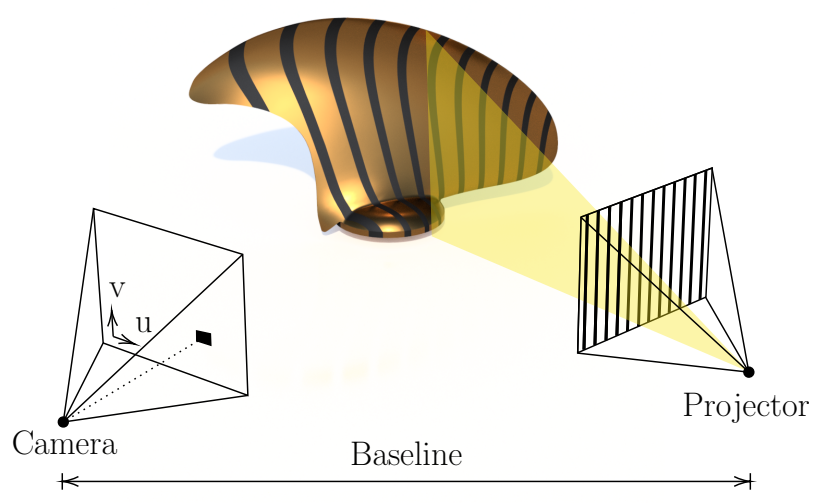

Figure 2: Working principle of structured light-based 3D cameras.

Then $\boldsymbol{R}$ is found by minimizing

$$
F_{2}=\sum_{j=1}^{m}\left\|\delta \boldsymbol{y}_{j}-\boldsymbol{R} \delta \boldsymbol{x}_{j}\right\|^{2}
$$

which is known as the Procrustes problem (Golub and Van Loan, 1996). The optimal solution is found by the maximization of $\operatorname{tr}(\boldsymbol{R H})$ where $\boldsymbol{H}=\boldsymbol{B} \boldsymbol{A}^{\mathrm{T}}$ and $\boldsymbol{A}=\left(\delta \boldsymbol{y}_{1}, \ldots, \delta \boldsymbol{y}_{m}\right)$ and $\boldsymbol{B}=\left(\delta \boldsymbol{x}_{1}, \ldots, \delta \boldsymbol{x}_{m}\right)$. The optimal solution is

$$
\boldsymbol{R}=\boldsymbol{V} \boldsymbol{S} \boldsymbol{U}^{\mathrm{T}}
$$

where the $\boldsymbol{U}$ and $\boldsymbol{V}$ matrices are given by the singular value decomposition

$$
\boldsymbol{H}=\boldsymbol{U} \boldsymbol{\Sigma} \boldsymbol{V}^{\mathrm{T}}
$$

where $\boldsymbol{S}=\operatorname{diag}\left[1,1, \operatorname{det}\left(\boldsymbol{V} \boldsymbol{U}^{\mathrm{T}}\right)\right]$ ensures that $\boldsymbol{R}$ is a rotation matrix. The translation is then found from

$$
\boldsymbol{t}=\overline{\boldsymbol{y}}-\boldsymbol{R} \overline{\boldsymbol{x}} .
$$

The calibration procedure above assumes the transformation between the camera frame and the robot end-effector to be constant. For systems with significant distance between the camera and robot endeffector, the transformation is likely to be influenced by deformations in the intermediate structure. When the acquired point clouds are combined into a single model, a non-constant displacement between the camera and robot end-effector introduces errors.

\subsection{Structured light principle}

A structured light camera system uses an active stereovision method, where a projector is used for illuminating an object by a sequence of known patterns. Simultaneously, the target object is observed by a camera sensor and stored as a 2D image of the scene. When the object is a planar surface, the perceived pattern will resemble the projected structured light pattern. However, if the observed object has nonplanar surfaces, the pattern perceived by the camera is distorted by the geometric shape of the object. As such, 3D depth information can be extracted by analyzing the disparity from the original projected pattern. The method is illustrated in Figure 2.

In order to extract the depth information, it is necessary to describe the relationship between the measured image coordinates and the 3D world coordinates. The camera sensor can be modeled with the coordinate frame $c$ fixed in the optical center of the camera, the $x_{c} y_{c}$-plane as the focal plane, and the $z_{c}$-axis as the optical axis pointing out of the camera lens and towards the target object. Let the position of a world point $P$ relative to the origin of frame $c$ be given by $\vec{r}$. The vector $\vec{r}$ given in the coordinates of frame $c$ is

$$
\boldsymbol{r}=\left[\begin{array}{l}
x \\
y \\
z
\end{array}\right], \quad \tilde{\boldsymbol{r}}=\left[\begin{array}{c}
x \\
y \\
z \\
1
\end{array}\right]
$$

where $\boldsymbol{r} \in \mathbb{R}^{3}$ is the Euclidean coordinate vector, and the tilde notation $\tilde{\boldsymbol{a}}$ is the corresponding homogeneous representation of a vector $\boldsymbol{a}$. In analogy with stereo vision techniques (Hartley and Zisserman, 2004), the camera and projector model is given by

$$
\begin{array}{cl}
\lambda_{1} \tilde{\boldsymbol{s}}_{1}=\boldsymbol{P}_{1} \tilde{\boldsymbol{r}}, & \lambda_{2} \tilde{\boldsymbol{s}}_{2}=\boldsymbol{P}_{2} \tilde{\boldsymbol{r}} \\
\tilde{\boldsymbol{p}}_{1}=\boldsymbol{K}_{1} \tilde{\boldsymbol{s}}_{1}, & \tilde{\boldsymbol{p}}_{2}=\boldsymbol{K}_{2} \tilde{\boldsymbol{s}}_{2}
\end{array}
$$

where the world point $P$ is mapped to the normalized image coordinates $\boldsymbol{s}_{1}$ of the camera, and the normalized coordinates $s_{2}$ of the projector. The pixel coordinates of the camera and projector are $\boldsymbol{p}_{1}$ and $\boldsymbol{p}_{2}$, respectively. $\lambda_{1}$ and $\lambda_{2}$ are depth factors, $\boldsymbol{K}_{1}$ is the camera parameter matrix, and $\boldsymbol{K}_{2}$ is the projector parameter matrix. $\boldsymbol{P}_{1}=[\boldsymbol{I} \mid \mathbf{0}]$ is the camera matrix and $\boldsymbol{P}_{2}=[\boldsymbol{R} \mid \boldsymbol{t}]$ is the projector matrix. It follows that the homogeneous normalized coordinates of the camera and projector are given by

$$
\begin{aligned}
& \lambda_{1} \tilde{\boldsymbol{s}}_{1}=\boldsymbol{r} \\
& \lambda_{2} \tilde{\boldsymbol{s}}_{2}=\boldsymbol{R} \boldsymbol{r}+\boldsymbol{t} .
\end{aligned}
$$

When the camera pixel coordinates $\boldsymbol{p}_{1}$ are determined for a light ray with projector pixel coordinates $\boldsymbol{p}_{2}$, the homogeneous normalized coordinates are found from $\tilde{\boldsymbol{s}}_{1}=\boldsymbol{K}_{1}^{-1} \tilde{\boldsymbol{p}}_{1}$ and $\tilde{\boldsymbol{s}}_{2}=\boldsymbol{K}_{2}^{-1} \tilde{\boldsymbol{p}}_{2}$.

A ray, or a line $\boldsymbol{l}_{1}$, is defined by the homogeneous normalized coordinate $\boldsymbol{s}_{1}$ and the camera center. Similarly, a line $\boldsymbol{l}_{2}$ is defined by $\boldsymbol{s}_{2}$ and the projector center. 
The two lines intersect in the world point $P$. This process is also known as triangulation (Hartley and Zisserman, 2004). The intersection between the lines $\boldsymbol{l}_{1}$ and $\boldsymbol{l}_{2}$ must be kept fixed during the exposure time needed for the camera to capture the sequence of patterns illuminating the object. Hence, it is a prerequisite that the target object and camera is at rest during the acquisition time.

Conventional structured light camera systems are capable of acquiring a scalar value, such as the reflectance of the surface, associated with each point on the surface of the target object. The scalar value $I_{k}$ for each surface point $\boldsymbol{r}_{k}=\left[x_{k}, y_{k}, z_{k}\right]^{T} \in \mathbb{R}^{3}$ of the acquired dataset is joined in the point cloud $\boldsymbol{P}_{k}=\left[x_{k}, y_{k}, z_{k}, I_{k}\right]^{T}, k=1, \ldots, N$. Also, scalar color intensity values $r_{k}, g_{k}, b_{k}$ can be acquired for each measured point and combined to the point cloud $\boldsymbol{P}_{k}=\left[x_{k}, y_{k}, z_{k}, I_{k}, r_{k}, g_{k}, b_{k}\right]^{T}, k=1, \ldots, N$.

\section{Input Shaping}

Suppose that a 3D camera is attached to the robot with a mechanical connection that is elastic in one direction. The model of the elastic interconnection is

$$
m \ddot{x}_{c}+c\left(\dot{x}_{c}-\dot{x}_{0}\right)+k\left(x_{c}-x_{0}\right)=0
$$

where $x_{c}$ is the position of the camera, $x_{0}$ is the position of the attachment point, $c$ is the damping coefficient and $k$ is the spring stiffness. This gives

$$
\ddot{x}+2 \zeta \omega_{0} x+\omega_{0}^{2} x=u
$$

where $x=x_{c}-x_{0}$ is the elastic deflection, $\omega_{0}=\sqrt{k / m}$ is the undamped natural frequency, $\zeta=c /(2 \sqrt{\mathrm{km}})$ is the relative damping, and $u=\ddot{x}_{0}$ is the acceleration of the attachment point. It is assumed that the system is underdamped with relative damping satisfying $0 \leq$ $\zeta \leq 1$. The Laplace transform of (22) gives

$$
H(s)=\frac{x(s)}{u(s)}=\frac{1}{s^{2}+2 \zeta \omega_{0} s+\omega_{0}^{2}}
$$

which has complex poles at $\lambda=\zeta \omega_{0} \pm j \omega_{d}$, where

$$
\omega_{d}=\omega_{0} \sqrt{1-\zeta^{2}}
$$

is the damped natural frequency. The impulse response $h(t)$ corresponding to $H(s)$ is found by the inverse Laplace transform, of (23), which gives

$$
h(t)=\frac{1}{\omega_{d}} e^{-\omega_{0} \zeta t} \sin \left(\omega_{d} t\right) .
$$

Input shaping, as described in Singer (1989), is designed using a description of the impulse response of the system. Define the unit pulse function as in Robinett et al. (2002) by

$$
\delta_{\Delta}(t)= \begin{cases}0, & t \leq 0 \\ 1 / \Delta, & 0 \leq t \leq \Delta . \\ 0, & t \geq \Delta\end{cases}
$$

Then the Dirac delta function $\delta(t)$, which is also called a unit impulse function, can be considered to be the limit of the unit impulse function when the pulse width tends to zero. This is written

$$
\delta(t)=\lim _{\Delta \rightarrow 0} \delta_{\Delta}(t) .
$$

It is noted that $\int_{-\infty}^{\infty} \delta(t) d t=1$, and that the Laplace Transform of the unit impulse function $\delta(t)$ is 1 .

The response to a unit impulse $u(t)=\delta\left(t-t_{i}\right)$ at $t=t_{i}$ is

$$
x(t)=h(t) * \delta\left(t-t_{i}\right)=h\left(t-t_{i}\right)
$$

where $*$ is the convolution operator.

As in Singer (1989), we consider the case where a sequence of $N$ impulse functions $A_{i} \delta\left(t-t_{i}\right)$ are input to the system, where $t_{1}<\ldots<t_{N}$. Then the response of the system for $t \geq t_{N}$ is given by

$$
\begin{aligned}
x(t) & =\sum_{i=1}^{N} A_{i} h\left(t-t_{i}\right) \\
& =\sum_{i=1}^{N} \frac{A_{i}}{\omega_{d}} e^{-\omega_{0} \zeta\left(t-t_{i}\right)} \sin \left(\omega_{d}\left(t-t_{i}\right)\right) \\
& =e^{-\omega_{0} \zeta t} \sum_{i=1}^{N} B_{i} \sin \left(\omega_{d} t-\phi_{i}\right)
\end{aligned}
$$

where

$$
B_{i}=\frac{A_{i}}{\omega_{d}} e^{\omega_{0} \zeta t_{i}} \quad \text { and } \quad \phi_{i}=\omega_{d} t_{i}
$$

Then, from the trigonometric identity

$$
\sin \left(\omega_{d} t-\phi_{i}\right)=\cos \left(\phi_{i}\right) \sin \left(\omega_{d} t\right)-\sin \left(\phi_{i}\right) \cos \left(\omega_{d} t\right)
$$

it follows that

$$
\sum_{i=1}^{N} B_{i} \sin \left(\omega_{d} t-\phi_{i}\right)=V_{1} \sin \left(\omega_{d} t\right)-V_{2} \cos \left(\omega_{d} t\right)
$$

where

$$
V_{1}=\sum_{i=1}^{N} B_{i} \cos \left(\phi_{i}\right) \quad \text { and } \quad V_{2}=\sum_{i=1}^{N} B_{i} \sin \left(\phi_{i}\right) .
$$

This can be written

$$
B \sin \left(\omega_{d} t+\phi\right)=V_{1} \sin \left(\omega_{d} t\right)-V_{2} \cos \left(\omega_{d} t\right)
$$


where

$$
B=\sqrt{V_{1}^{2}+V_{2}^{2}} \text { and } \phi=-\arctan \left(V_{2} / V_{1}\right)
$$

and it is seen that the response can be written ( $\mathrm{Yu}$ Zhao et al., 2016)

$$
x(t)=e^{-\omega_{0} \zeta t} B \sin \left(\omega_{d} t+\phi\right), \quad t \geq t_{N} .
$$

This means that $B=0$ is achieved by selecting

$$
V_{1}=V_{2}=0
$$

This results in $x(t)=0$ for $t \geq t_{N}$, which means that the elastic deflection is zero after time $t_{N}$.

Generation of the sequence $A_{i} \delta\left(t-t_{i}\right)$ of input impulses is the next issue to be discussed. Suppose that the attachment point $x_{0}$ is to be positioned at the desired point $x_{d}$, and that a reference trajectory for the acceleration is generated as $\ddot{x}_{0}(t)=u_{r}(t)$ for $0 \leq t \leq t_{f}$, where $u_{r}(t)$ is selected so that $x_{0}(t)=x_{d}$ for $t \geq t_{f}$. Suppose that $N=2$, and that $A_{1}$ and $A_{2}$ are positive. Then

$$
\begin{aligned}
u(t) & =u_{r}(t) * A_{1} \delta(t)+u_{r}(t) * A_{2} \delta\left(t-t_{2}\right) \\
& =A_{1} u_{r}(t)+A_{2} u_{r}\left(t-t_{2}\right) .
\end{aligned}
$$

This will result in $x_{0}(t)=\left(A_{1}+A_{2}\right) x_{d}$ for $t \geq t_{f}+$ $t_{2}$, which means that if the constants $A_{1}$ and $A_{2}$ are selected so that

$$
A_{1}+A_{2}=1
$$

then

$$
x(t)=0 \text { and } x_{0}(t)=x_{d} \text { for } t \geq t_{f}+t_{2} .
$$

It has now been established that if $V_{1}$ and $V_{2}$ are selected according to (36) and $A_{1}$ and $A_{2}$ are selected according to (39), then the desired position $x_{0}=x_{d}$ is achieved with zero elastic deflection for $t \geq t_{f}+t_{2}$. This approach is known as input shaping (Kamel et al., 2008). It is noted that any unshaped command $u_{r}(t)$ can be used for the desired acceleration of the endeffector.

\section{$3.1 \mathrm{ZV}$}

In Robinett et al. (2002); Singer (1989); Yu Zhao et al. (2016); Kamel et al. (2008), a zero vibration (ZV) input shaper can be given as

$$
f_{\mathrm{ZV}}(t)=\sum_{i=1}^{2} A_{i} \delta\left(t-t_{i}\right)
$$

a sequence of two impulses $A_{1} \delta\left(t-t_{1}\right)$ and $A_{2} \delta\left(t-t_{2}\right)$. To design a ZV shaping filter we need to derive the amplitudes $A_{1}$ and $A_{2}$ and the associated time instants $t_{1}$ and $t_{2}$. The constraints

$$
t_{2}>t_{1} \text { and } A_{i}>0
$$

are used. By superposition, the system response for $t \geq t_{2}$ is seen to be

$$
\begin{aligned}
x(t) & =f_{\mathrm{ZV}}(t) * h(t)=\sum_{i=1}^{2} A_{i} h\left(t-t_{i}\right) \\
& =K e^{-\omega_{0} \zeta t} B \sin \left(\omega_{d} t+\phi\right)
\end{aligned}
$$

where the shifted impulse response $h\left(t-t_{i}\right)$ is given in (28) and the amplitude $B$ and phase delay $\phi$ are given in (34). The terms $V_{1}$ and $V_{2}$ in the amplitude of the vibration $B=\sqrt{V_{1}^{2}+V_{2}^{2}}$ are from (32) seen to be

$$
V_{1}=\sum_{i=1}^{2} B_{i} \cos \left(\phi_{i}\right) \quad \text { and } \quad V_{2}=\sum_{i=1}^{2} B_{i} \sin \left(\phi_{i}\right) \text {. }
$$

Elimination of camera vibration after the last (second) impulse has occurred requires that the amplitude $B$ is equal to zero at the time which the second impulse ends $t>t_{2}$ (Singer, 1989). That is, we want both $V_{1}$ and $V_{2}$ to be $V_{1}=V_{2}=0$ because they are squared in (34) (Robinett et al., 2002; Singh and Singhose, 2002). In addition, if we want the shaped command to give the same result as the unshaped command, then the sum of the two amplitudes of the impulses should be

$$
A_{1}+A_{2}=1
$$

which is referred to as unity static gain (Kamel et al., 2008). Without loss of generality, the time instant $t_{1}$ can be zero, $t_{1}=0$. We want $V_{2}=0$, and it is seen that by inserting for $t_{1}=0$ in (44), we get

$$
V_{2}=B_{2} \sin \left(\phi_{2}\right)=0
$$

where it is used that $B_{1} \sin \left(\phi_{1}\right)=B_{1} \sin \left(\omega_{d} t_{1}\right)=0$, which is obtained by inserting for $t_{1}=0 \mathrm{in}(30)$. The constraint in (46) can be satisfied when we choose $t_{2}$ such that $\sin \left(\phi_{2}\right)=\sin \left(\omega_{d} t_{2}\right)=0$, which occurs when

$$
\phi_{2}=\omega_{d} t_{2}=n \pi \Rightarrow t_{2}=\frac{n \pi}{\omega_{d}}, n \in \mathbb{N}-\{0\} .
$$

Naturally, we want to cancel the vibration in the shortest possible time, such that we can set $t_{2}=\pi / \omega_{d}$. Next, in order to also get $V_{1}=0$, we substitute $A_{2}=1-A_{1}, t_{1}=0, t_{2}=\pi / \omega_{d}, \phi_{2}=\omega_{d} t_{2}=\pi$ into $V_{1}$ in (44), which gives

$$
\begin{aligned}
V_{1} & =B_{1}+B_{2} \cos \left(\phi_{2}\right) \\
& =B_{1}-B_{2} \\
& =\frac{\omega_{0}^{2}}{\omega_{d}}\left[A_{1}-A_{2} e^{\omega_{0} \zeta t_{2}}\right] .
\end{aligned}
$$


We introduce a constant

$$
k=e^{-\omega_{0} \zeta t_{2}}=e^{-\frac{\omega_{0} \zeta \pi}{\omega_{d}}}=e^{-\frac{\zeta \pi}{\sqrt{1-\zeta^{2}}}}
$$

where it is seen that $e^{\omega_{0} \zeta t_{2}}=1 / k$. By applying this and reformulating (45) to $A_{2}=1-A_{1}$, we have from (48) that $V_{1}=0$ when

$$
A_{1}=\frac{1-A_{1}}{k} \rightarrow A_{1}=\frac{1}{1+k}
$$

and consequently

$$
A_{2}=1-A_{1}=\frac{k}{k+1} .
$$

The $Z V$-shaper can then be summarized as (Singh and Singhose, 2002; Zhao and Tomizuka, 2017)

$$
\left[\begin{array}{c}
A_{i} \\
t_{i}
\end{array}\right]=\left[\begin{array}{cc}
\frac{1}{1+k} & \frac{k}{1+k} \\
0 & \frac{\pi}{\omega_{d}}
\end{array}\right]
$$

This shaper will give zero residual vibration if the natural frequency $\omega_{0}$ and damping ratio $\zeta$ are known precisely (Singer and Seering, 1990).

\subsection{ZVD}

The amplitudes $A_{i}$ and time instances $t_{i}$ of the impulses depend on the system's natural frequency $\omega_{0}$ and damping ratio $\zeta$. In order to add robustness to the system after the input has ended, additional constraints can be added. These constraints provide a more robust shaper in the case of modeling error due to imprecise identification of the system's true natural frequency and damping ratio.

The Zero Vibration and Derivative (ZVD) shaper is described by three impulses $A_{i} \delta\left(t-t_{i}\right)$ for $i=[1,2,3]$ as

$$
f_{\mathrm{ZVD}}(t)=\sum_{i=1}^{3} A_{i} \delta\left(t-t_{i}\right)
$$

unlike the ZV shaper which was described by two impulses. The response of the system to three impulses is seen to be

$$
\begin{aligned}
x(t) & =f_{\mathrm{ZV} D}(t) * h(t)=\sum_{i=1}^{3} A_{i} h\left(t-t_{i}\right) \\
& =K e^{-\omega_{0} \zeta t} B \sin \left(\omega_{d} t+\phi\right)
\end{aligned}
$$

where the amplitude $B$ is

$$
B=\sqrt{V_{1}^{2}+V_{2}^{2}}
$$

where

$$
V_{1}=\sum_{i=1}^{3} B_{i} \cos \left(\phi_{i}\right) \text { and } V_{2}=\sum_{i=1}^{3} B_{i} \sin \left(\phi_{i}\right) .
$$

As for the ZV shaper, $B=0$ for $V_{1}=V_{2}=0$ (Section 3.1). The additional constraints, which make it a ZVD shaper, are formed by requiring that the derivatives of $V_{1}$ and $V_{2}$ with respect to $\zeta$ and $\omega_{0}$ are equal to zero. It was shown in Singer (1989) that if the derivatives with respect to $\omega_{0}$ are zero, then the derivatives with respect to $\zeta$ will be zero. Therefore, robustness to errors in $\zeta$ has been obtained by solving for robustness to errors in $\omega_{0}$. We then have the constraints for eliminating residual oscillation

$$
\begin{aligned}
& V_{1}=\sum_{i=1}^{3} B_{i} \cos \left(\phi_{i}\right)=0 \\
& V_{2}=\sum_{i=1}^{3} B_{i} \sin \left(\phi_{i}\right)=0
\end{aligned}
$$

and the constraints related to errors in the estimate of $\omega_{0}$ and $\zeta$ is from Singer (1989); Singer and Seering (1990); Robinett et al. (2002) given as

$$
\frac{\partial V_{1}}{\partial \omega_{0}}=0 \quad \text { and } \quad \frac{\partial V_{2}}{\partial \omega_{0}}=0
$$

The solution of (59) can be proven to be

$$
\begin{aligned}
& \sum_{i=1}^{3} B_{i} t_{i} \cos \left(\phi_{i}\right)=0 \\
& \sum_{i=1}^{3} B_{i} t_{i} \sin \left(\phi_{i}\right)=0 .
\end{aligned}
$$

The procedure for determining the parameters $A_{i}$ and $t_{i}$ is to set $A_{1}=1$ and $t_{1}=0$ initially, and then solve for $A_{2}, A_{3}, t_{2}$ and $t_{3}$ using the constraints $(57,58,60,61)$ (Singer, 1989). The amplitude parameters are then normalized so that $A_{1}+A_{2}+A_{3}=1$. This gives

$$
\left[\begin{array}{c}
A_{i} \\
t_{i}
\end{array}\right]=\left[\begin{array}{ccc}
\frac{1}{1+2 k+k^{2}} & \frac{2 k}{1+2 k+k^{2}} & \frac{k^{2}}{1+2 k+k^{2}} \\
0 & \frac{\pi}{\omega_{d}} & \frac{2 \pi}{\omega_{d}}
\end{array}\right]
$$

\section{$3.3 \mathrm{EI}$}

The ZV and ZVD shapers are designed with hard constraints for which the goal is to have zero residual vibration. The real camera system might slightly differ from the mathematical description in (22), and some residual vibration could occur even at the true natural frequency. Therefore, it could be of interest to relax 
the hard constraints in Sections 3.1 and 3.2 and allow for some small residual vibration. For a type of shapers, called Specified Insensitivity (SI) shapers, the constraints are relaxed in that they vary with the desired level of insensitivity (Singhose et al., 1996). An example of this type of shaper is the Extra Insensitive shaper (EI). The EI shaper also has three impulses

$$
f_{\mathrm{EI}}(t)=\sum_{i=1}^{3} A_{i} \delta\left(t-t_{i}\right)
$$

as for the ZVD shaper but with a different time instance $t_{2}$ and amplitudes $A_{1}, A_{2}$ and $A_{3}$. The EI shaper is from Singhose et al. (1994) given as

$$
\left[\begin{array}{c}
A_{i} \\
t_{i}
\end{array}\right]=\left[\begin{array}{ccc}
A_{1} & A_{2} & A_{3} \\
0 & t_{2} & \frac{2 \pi}{\omega_{d}}
\end{array}\right]
$$

where

$$
\begin{aligned}
A_{1}= & 0.2497+0.2496 V_{t o l}+0.8001 V_{t o l}+1.233 V_{t o l} \zeta \\
& +0.4960 \zeta^{2}+3.173 V_{t o l} \zeta^{2} \\
A_{2}= & 1-\left(A_{1}+A_{3}\right) \\
A_{3}= & 0.2515+0.2147 V_{t o l}-0.8325 \zeta+1.415 V_{t o l} \zeta \\
& +0.8518 \zeta^{2}-4.901 V_{t o l} \zeta^{2} \\
t_{2}= & {\left[0.5+0.4616 V_{t o l} \zeta+4.262 V_{t o l} \zeta^{2}+1.756 V_{t o l} \zeta^{3}\right.} \\
& \left.+8.578 V_{t o l}^{2} \zeta-108.6 V_{t o l}^{2} \zeta^{2}+337 V_{t o l}^{2} \zeta^{3}\right] t_{3},
\end{aligned}
$$

and where $V_{t o l}$ is the tolerable level of vibration (Vaughan et al., 2008).

\section{Experiments}

\subsection{Input shapers}

The commanded input shaped acceleration is

$$
u(t)=f(t) * u_{r}(t)=\sum_{i=1}^{N} A_{i} u_{r}\left(t-t_{i}\right)
$$

where $f(t)$ is the input shaper, and $u_{r}$ is the desired reference of the acceleration. The input shaper $f(t)$ of the type ZV, ZVD, and EI are given in (41), (53), and (63), respectively. Assuming that the desired reference trajectory is a trapezoidal velocity profile without a cruise velocity segment, the desired acceleration can be given as described in Siciliano et al. (2008)

$$
u_{r}(t)= \begin{cases}a, & 0 \leq t \leq t_{c} \\ 0, & t_{c}<t \leq t_{f}+t_{c} \\ -a, & t_{f}+t_{c}<t \leq t_{f} \\ 0, & t_{f}<t\end{cases}
$$

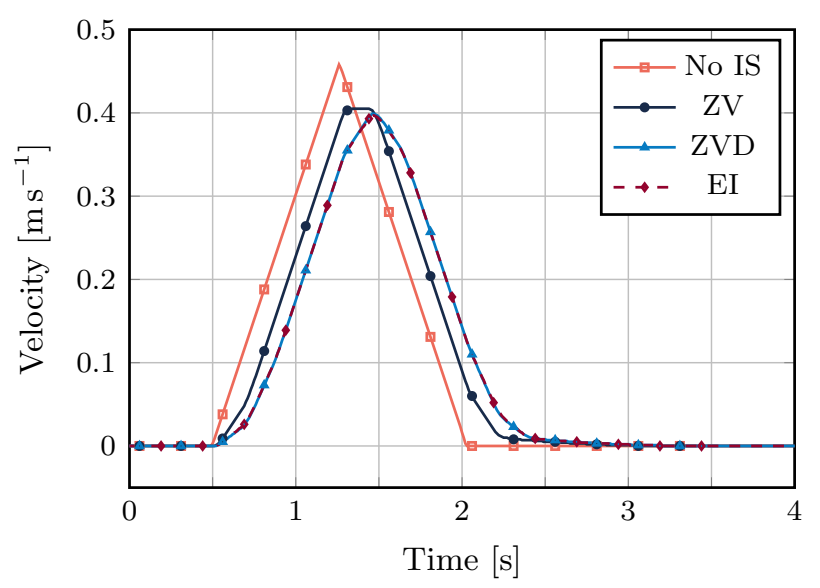

Figure 3: Comparison of driven velocity profiles $v(t)$ for the various shapers.

where $t_{f}$ is the final time of the trajectory, $t_{c}$ is the time interval of a parabolic segment with constant acceleration $a$. The constant acceleration $a$ is a design parameter while

$$
\begin{aligned}
t_{f} & =\sqrt{4\left(x_{0}^{f}-x_{0}^{i}\right) / a} \\
t_{c} & =\left(t_{f}-\sqrt{t_{f}^{2}-t_{f}}\right) / 2
\end{aligned}
$$

and where $x_{0}^{i}$ and $x_{0}^{f}$ are the initial and final desired positions of the attachment point $x_{0}$.

It is not feasible to command the acceleration of the attachment point directly in a robot controller. Therefore, it is proposed to use a velocity loop modeled as a velocity loop for electrical actuators to get a velocity command, which is further used as input to the robot controller (Rauscher et al., 2018). This was achieved in the experiments by integrating the acceleration command $u$ to the velocity command $w$ and using it as input to the velocity loop. The model used is given by

$$
\begin{aligned}
\dot{w} & =u \\
\dot{v} & =\frac{1}{T_{v}}(w-v)
\end{aligned}
$$

where $1 / T_{v}$ is the bandwidth of the velocity loop. The driven velocity $v$ was used as a velocity input to the robot controller. The driven acceleration $\dot{v}$ is expected to be close to the commanded acceleration $u$ if the chosen bandwidth $1 / T_{v}$ is faster or close to the bandwidth of the shaper. The driven velocity profiles for the different shapers are illustrated in Figure 3.

\subsection{Experimental setup}

The measurement system consists of a Universal Robots UR10 articulated robot manipulator equipped 


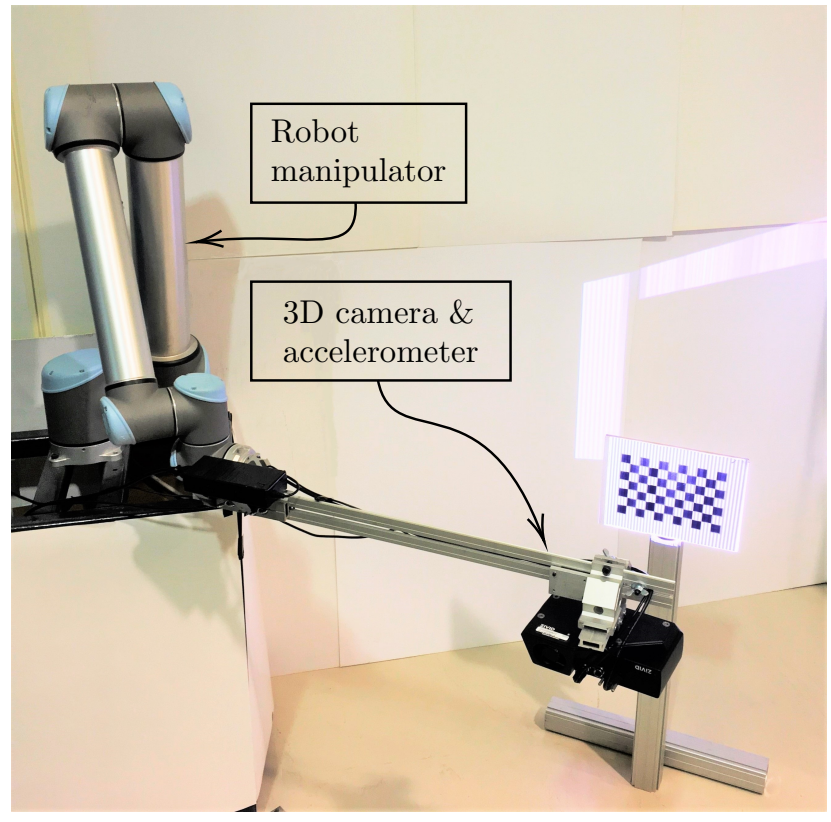

Figure 4: The experimental setup for comparison of the shapers.

with a Zivid 3D-camera. An accelerometer is attached next to the camera for monitoring vibrations. The robot has a maximum reach of $1300 \mathrm{~mm}$, and a rated payload capacity of $10 \mathrm{~kg}$. The $3 \mathrm{D}$-camera captures depth data at an imaging resolution of $1920 \times 1200$ pixels at a rate of $10 \mathrm{~Hz}$. The camera offers high-dynamicrange imaging (HDR) capabilities and has a field of view that is $780 \times 490 \mathrm{~mm}$ at a scanning distance of $1.1 \mathrm{~m}$, whereas the working distance of the camera is between $0.6 \mathrm{~m}$ and $1.1 \mathrm{~m}$. The range of the robot combined with the camera sensor field of view restricts the permissible extent of objects to be scanned accordingly. A naive approach for extending the scanning reach of the system is to lengthen the distance between the camera and robot end-effector by connecting the camera and robot via a fixed rod. An extruded aluminum profile with high stiffness and low weight is chosen due to the robot's payload capacity. The profile has a rectangular cross-section and lower stiffness in the direction of motion, increasing the camera settling times with unshaped robot trajectories. The system is illustrated in Figure 4.

Despite the camera acquisition rate of $10 \mathrm{~Hz}$, rapid motions while capturing causes inaccurate depth measurements. For each depth capture, several patterns are projected and recorded, and certain exposure time is needed to acquire each frame. When the 3D capturing is spread over multiple frames, the motion of the camera or the scanned object leads to distortion artifacts. The motion leads to a violation of the as- sumption that corresponding pixels in the captured images are depicting the same surface point. When capturing highly specular metal surfaces, such as that of propeller blades, the surface reflectivity is a challenging issue for structured light means. The 3D measurement accuracy is affected by the presence of overand under-exposed image regions, leading to significant difficulties in extracting the degraded projected patterns in such regions. With high-dynamic-range imaging (HDR), several narrow range exposures of the same object are combined into a single capture. Hence, the pattern is better preserved, and the underlying surface is more easily extracted in shadowed and saturated regions. The HDR technology nevertheless places even more stringent requirements for the camera to be at rest for accurate imaging.

The camera mass relative to the mass of the rod raises the flexibility of the system, resulting in substantial residual vibrations of the camera after each robot motion. The residual vibrations increase with higher robot velocity and accelerations, making it difficult to simultaneously perform fast and precise measurements, as it becomes necessary to introduce a delay after each robot motion for the camera to become sufficiently at rest before capturing the object surface. By convolving the desired motion signal with a proper input shaper, the system can respond smoothly without vibration from the input command.

We implemented and tested various input shapers in the described robotic measurement system, aiming to reduce and counteract the residual vibrations and thereby decrease the required settling-time following each robot motion. If the camera reaches a stationary state in a shorter period, the overall scanning time can be reduced.

\subsection{Parameter identification}

The dynamic model of the elastic deflection was experimentally validated by using an IMU for measuring the oscillations of the elastic deflection. The measurements from this experiment were compared to a simulated response using the model (22) with relative damping $\zeta=9 \times 10^{-3}$ and undamped natural frequency $\omega_{0}=17.6 \mathrm{rads}^{-1}$. Both the simulated and measured free decaying vibration of the camera system are shown in Figure 6. It is seen that the identified relative damping and natural frequency are close to the true system parameters. In the time interval $[2 \mathrm{~s}, 3 \mathrm{~s}$, there are some deviations between the identified and real model. The reason for this could be that the real model was slightly different from a linear second-order model. 


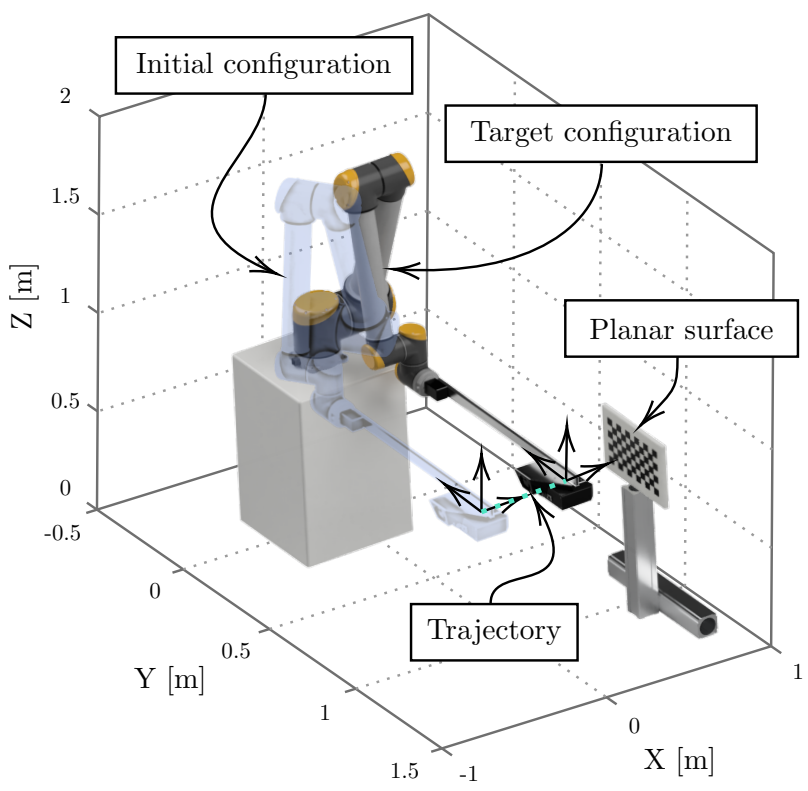

Figure 5: Robot path for the first experiment.

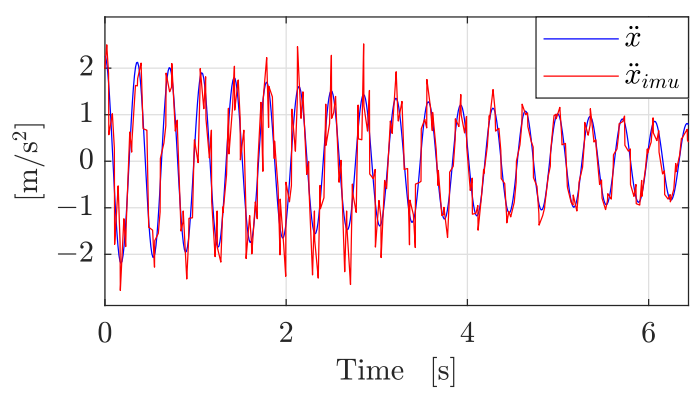

Figure 6: The simulated $\ddot{x}_{c}$ and measured $\ddot{x}_{i m u}$ acceleration of the camera due to decaying free vibration.

\subsection{Cases}

Two experiments were conducted to evaluate the different input shapers. First, a standard position increment was performed while the vibration damping of the different input shapers was recorded. For each run, a diffuse planar surface was scanned, and the quality of the scan was evaluated from 3D depth data captured by the camera. The scanning started immediately after a robot motion towards the surface had been performed. Additionally, the vibration response was recorded from the accelerometer. We evaluated the three shapers described in Section 3 for our scanning setup. A quantitative evaluation of the shapers is performed using the reference path shown in Figure 5, where the trajectory with the position, velocity, and acceleration profiles are given in Figure 7.
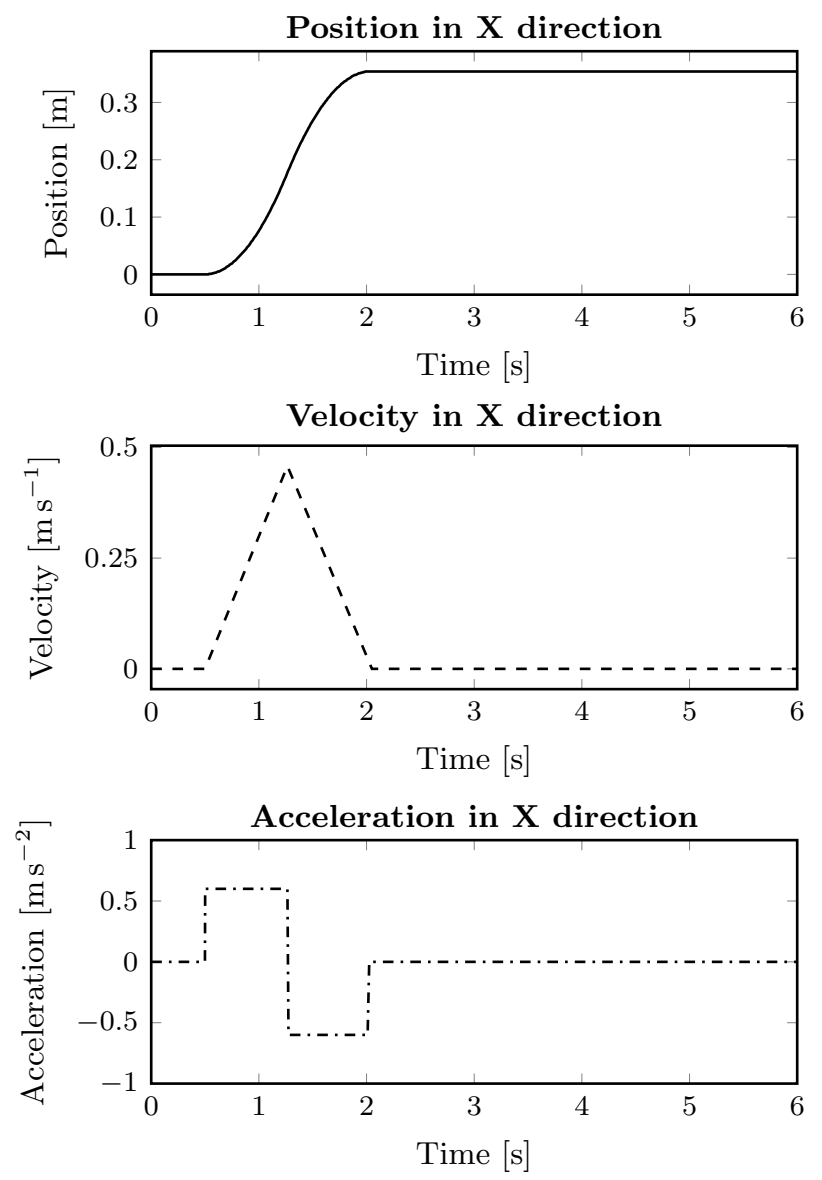

Figure 7: Reference trajectory for the first experiment. Position, velocity, and acceleration profiles along the Cartesian $x$-axis. 

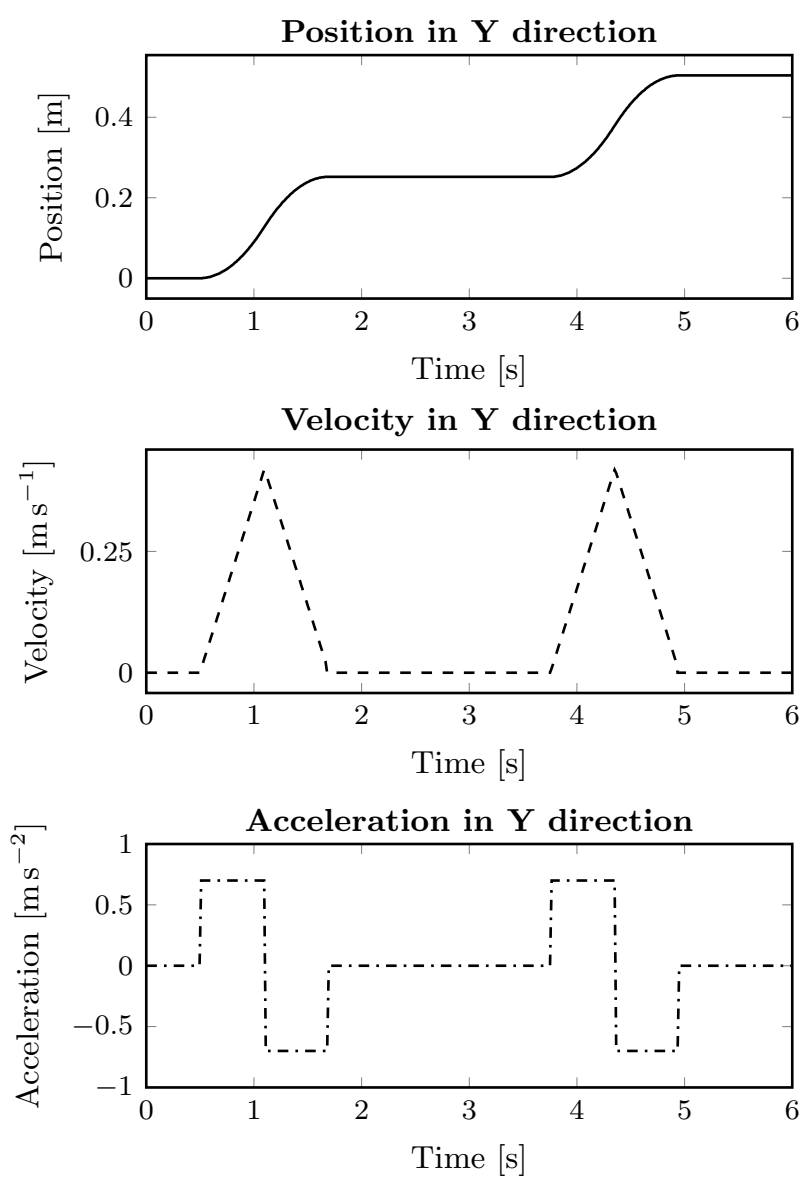

Figure 8: Trajectory for grabbing two 3D depth images of a propeller blade. The trajectory is performed along the Cartesian $y$-axis.

In our second experiment, we performed a practical $3 \mathrm{D}$ scanning of a marine propeller blade using the measurement system. The intent was to investigate and present the performance of the approach for a practical 3D scanning application. For this example, two capturing poses were needed to cover the blade surface. The robot trajectory for the second experiment is given in Figure 8.

\section{Experimental results and comparison}

The vibration responses for the three different input shapers, as measured by the accelerometer, are shown in Figure 9, where also the response for the case of unshaped motion is shown. The optimal tolerable level for the EI shaper was found to be $V_{t o l}=1 \%$ for the experimental case. It can be seen in the figure that all three input shapers significantly reduce residual vibra- tions compared to the unshaped case. However, the ZV shaper gave some residual vibration, which lasted for some seconds. As for the ZVD and EI shaper, it can be seen that the performance was almost identical. The vibrations were canceled at the same time instance for ZVD and EI. The EI shaper gave close to zero residual vibration, while ZVD had some minor residual vibration.

The measured depth values recorded by the 3Dcamera show the usefulness of the different shapers evaluated on a per-pixel basis. For each shaper, we sample 50 depth frames starting directly after the associated robot motion is completed. Additionally, we capture and evaluate the same amount of depth frames for the case of unshaped robot motion and a camera at rest. The resulting mean, standard deviation, and a Root-Mean-Square Error (RMSE) of the 50 frames are then calculated for each camera pixel. The result is shown as color frame plots in Figure 10, where the mean, standard deviation, and RMSE are the left, center, and right columns, respectively. As a reference for the RMSE evaluation, we make a RANSAC fit of a plane to an averaged sequence of 50 frames of the planar surface captured while the camera and robot are at rest. The resulting fit is a reference depth image $F_{\text {ref }}$. The RMSE is then computed for each depth pixel $(u, v)$ over the 50 frames with respect to a RANSAC plane fit of the planar surface as

$$
F_{\mathrm{RMSE}}(u, v)=\sqrt{\frac{1}{50} \sum_{i=1}^{50}\left(F_{i}(u, v)-F_{\mathrm{ref}}(u, v)\right)^{2}} .
$$

From the per-pixel evaluation, as illustrated in Figure 10, all three input shaping approaches have a beneficial impact on depth accuracy compared to the case with no input shaping. The ZVD and EI shapers display slightly better performance than the $\mathrm{ZV}$, while ZVD and EI show comparable performance. The effect is also noticeable from Figure 11, which shows the histogram distribution of the measured depth values of the $3 \mathrm{D}$-camera for the various shapers.

If the acquisition speed of the $3 \mathrm{D}$ scanning of objects is to be increased, the time delay required before each capture should be as low as possible. So, to accurately capture the surface of objects, the duration of the residual vibrations must be short while guaranteeing accurate positioning of the camera. As an assessment of how the depth measurement errors progress over time, we calculate an error metric from depth data captured at a rate of $10 \mathrm{~Hz}$ starting directly after a completed robot motion. A per-frame comparison of the RMSE for the three shapers is given in Figure 12. This RMSE is computed for each frame $F_{i}$ with respect to the RANSAC plane fit of the planar surface 

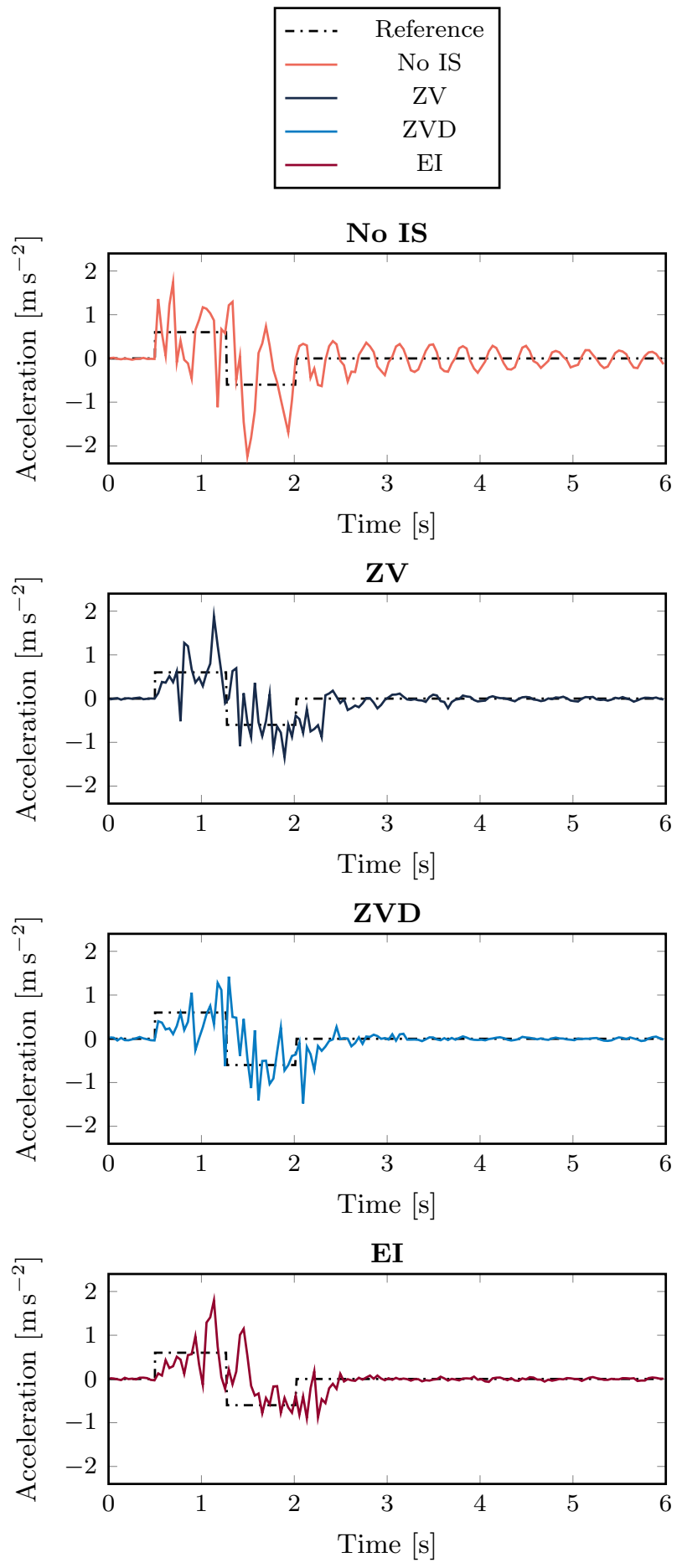

Figure 9: Acceleration measurements performed by an accelerometer placed right next to the $3 \mathrm{D}$ camera. Three different shapers are evaluated for the reference trajectory shown in Figure 7. as

$$
\mathrm{RMSE}=\sqrt{\frac{1}{n m} \sum_{u=1}^{n} \sum_{v=1}^{m}\left(F_{i}(u, v)-F_{\mathrm{ref}}(u, v)\right)^{2}}
$$

where $n$ and $m$ represent the width and height of the depth image pixel grid, and the reference values $F_{\text {ref }}(u, v)$ are determined from the plane fit of frames captured when the camera is at rest as above. It can be seen that all the shapers have a significant impact on the RMSE, with ZVD and EI giving the most stable results in terms of eliminating residual vibrations that might occur in a robotic $3 \mathrm{D}$ scanning operation. Since the ZVD shaper does not require any tuning, it is found to be the most favorable choice as a shaper for the second experiment.

In the second experiment, a marine propeller was scanned. This was achieved by recording $3 \mathrm{D}$ scan data along a reference trajectory performed by the robot. The robot trajectory is shown in Figure 8, where it is seen that the robot is kept stationary, for a short while, at two different positions. While the robot is held still, point clouds are grabbed from the 3D camera and stored. The resulting 3D scan, also represented as a point cloud, was obtained by merging the two sampled point clouds. The procedure was conducted for two cases, first with an unshaped reference trajectory and secondly with a ZVD shaped reference trajectory. The acceleration response of the camera as caused by the trajectories are shown in Figure 13. The resulting 3D scan data obtained with the unshaped and ZVD shaped trajectories are shown in Figures 14a and 14b, respectively. For the case where no shaper was used, the sampled point clouds did not align accurately due to the vibrations experienced by the camera while capturing. The vibration also caused significant levels of noise in the sampled point clouds. As described in Section 2.3 , it is a prerequisite that the target object and camera is at rest when the surface geometry is captured. Noise is introduced if the camera moves during the exposure time (Figure 14a). For the case with a ZVD shaper, the point clouds aligned more accurately and contained significantly less noise (Figure 14b).

\section{Conclusions}

This paper presents a comparison between three different input shapers adapted for a robotic measurement system for 3D scanning of large objects. The input shapers evaluated were the Zero Vibration (ZV), ZV Derivative (ZVD), and Extra Insensitive (EI) shapers. Using the input shapers, we have presented an approach for reducing residual vibrations, and thus, 


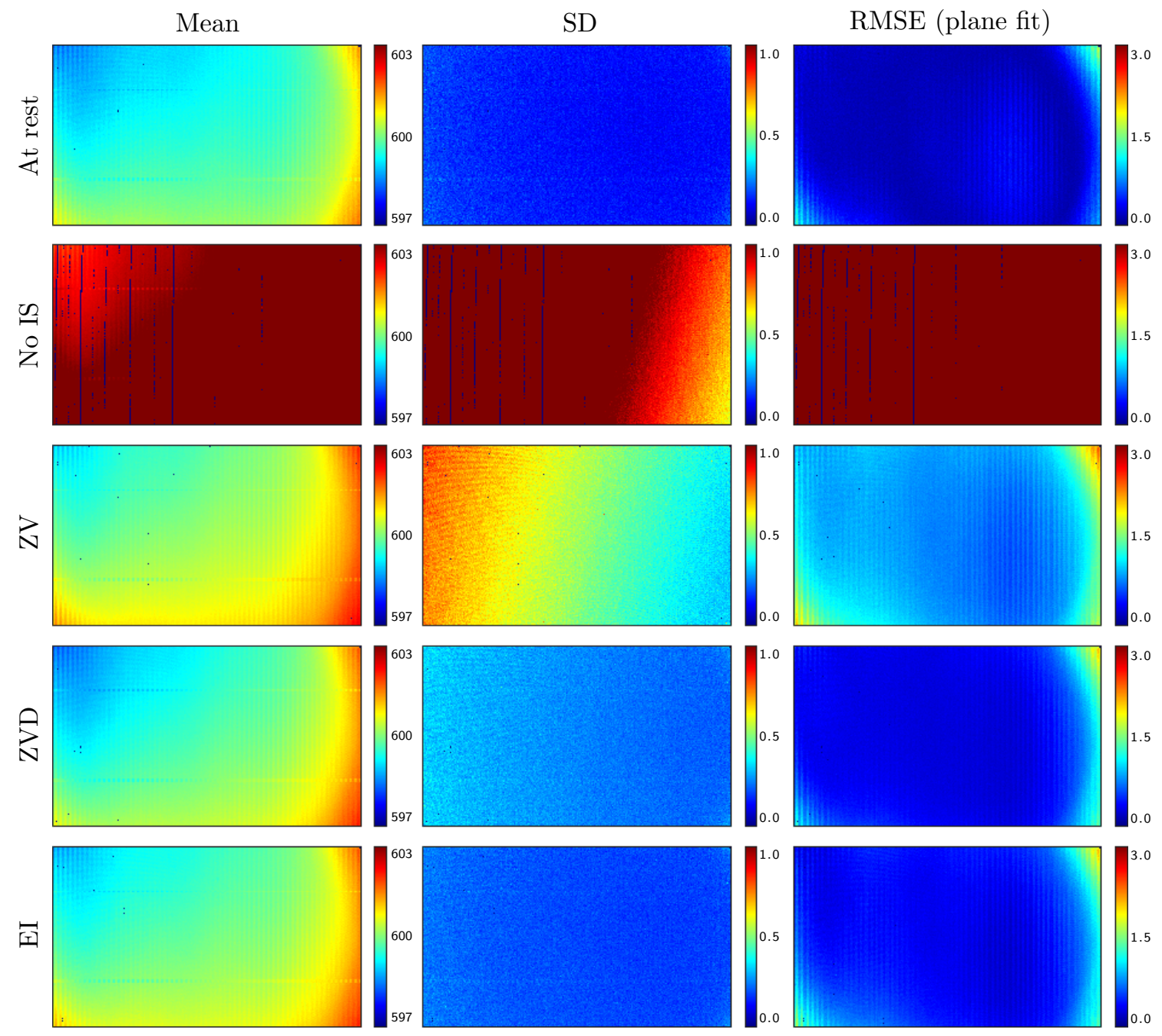

Figure 10: Statistics of 50 frames acquiring a planar surface (values in mm): Mean (left col.), standard deviation (middle col.), and RMSE with respect to a plane fitted using RANSAC (right col.). The 1st row is the raw range data captured with the robot at rest, and the 2nd row was captured when the robot executed the original, unshaped trajectory. For the 3rd row, the ZV shaper was used, the 4th row the ZVD shaper, and for the 5th, the EI shaper. 


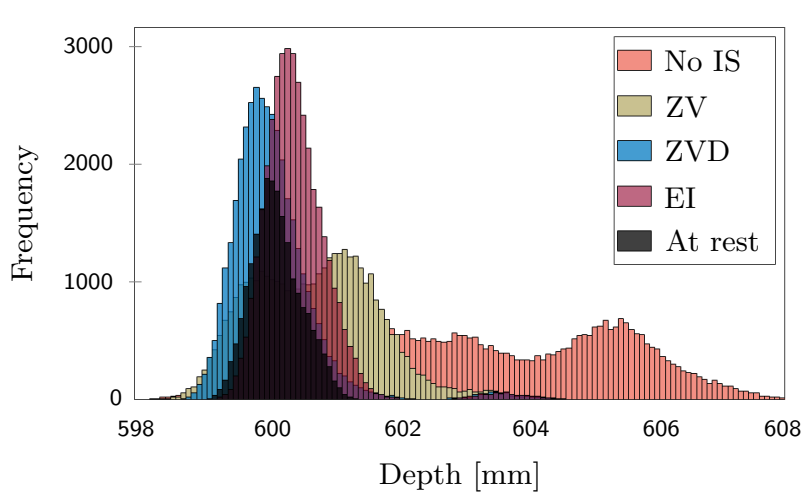

Figure 11: Histogram of depth pixel values for the various shapers during 50 frames, acquiring a planar surface.

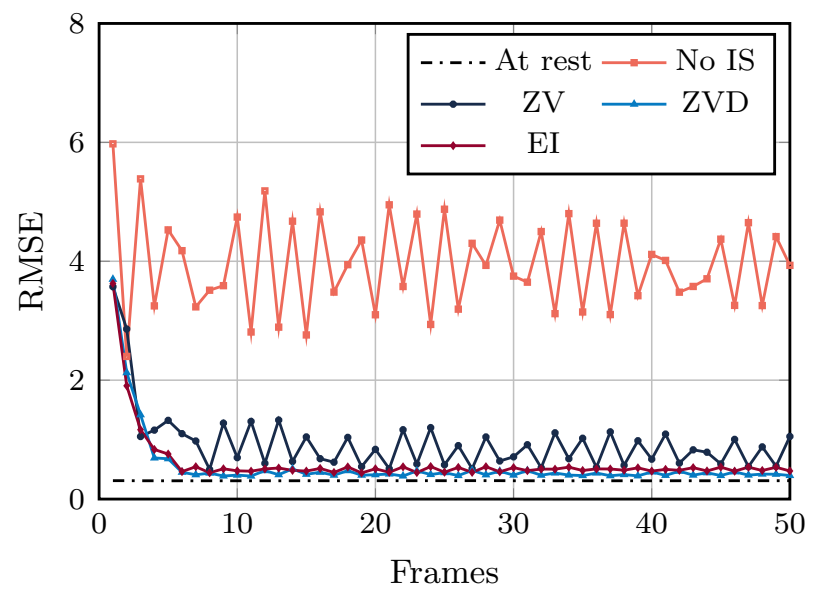

Figure 12: RMSE for the various shapers for the 50 first frames captured after the robot has reached the capturing position following the movement. The RMSE for 50 frames captured when the robot is at rest is given for reference.
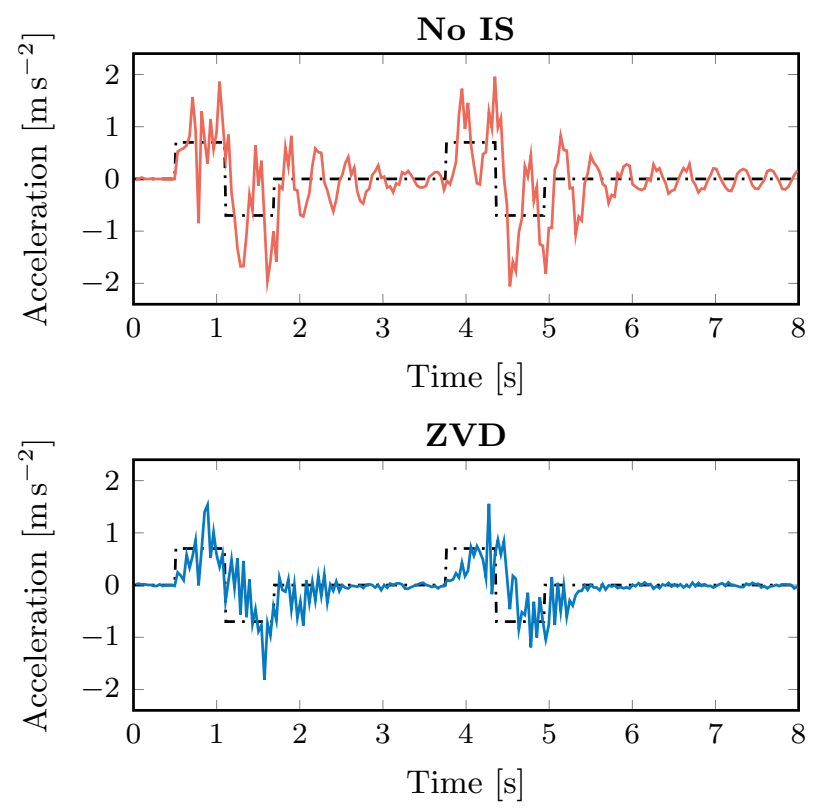

Figure 13: Acceleration measurements performed by an accelerometer placed right next to the $3 \mathrm{D}$ camera. The trajectory is performed in order to grab two 3D depth images of a ship propeller blade. The reference trajectory is shown in Figure 8.

achieving complete 3D scanning of the target objects in a shorter time.

Our evaluation was performed through two experiments. First, we used a 3D camera to capture depth data on a planar surface following a completed robot motion. Accelerometer readings for the three input shapers were obtained during the process. An extensive analysis of the captured data was performed, were the ZVD and EI shapers proved to give a consistent performance for the scanning application scenario. In the second experiment, a marine propeller blade was $3 \mathrm{D}$ scanned by recording scan data along a reference trajectory performed by the robot. Two different situations were tested one with an unshaped reference trajectory and one with a ZVD shaped trajectory. The resulting 3D scan generated from the ZVD shaped reference trajectory demonstrated to be superior compared to the case with an unshaped reference trajectory.

Future work will be focused on implementing input shaper for scanning directions spanning multiple degrees of freedom. 


\section{Acknowledgments}

The authors wish to thank the industrial partner Oshaug Metall AS of the OLIVER project. The project is funded by the Norwegian Research Council under the BIA program, project number 261639.

\section{References}

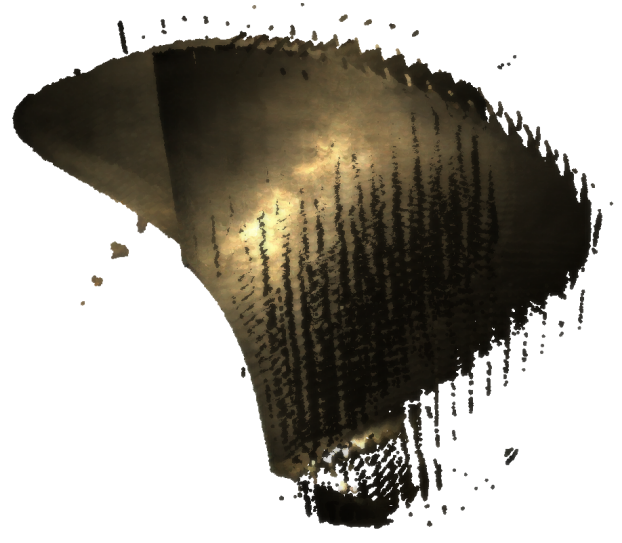

(a)

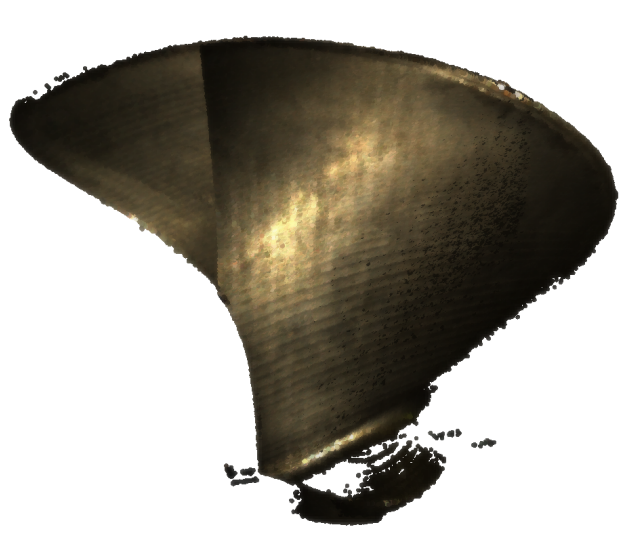

(b)

Figure 14: Point clouds of the propeller blade acquired using the unshaped reference trajectory (a) and the ZVD shaped trajectory (b).
Chen, F., Brown, G. M., and Song, M. Overview of three-dimensional shape measurement using optical methods. Optical Engineering, 2000. 39(1):10. doi:10.1117/1.602438.

Daniilidis, K. Hand-eye calibration using dual quaternions. The International Journal of Robotics Research, $1999 . \quad$ 18(3):286-298. doi:10.1177/02783649922066213.

Golub, G. H. and Van Loan, C. F. Matrix Computations. Johns Hopkins University Press, Baltimore, MD, USA, 3rd edition, 1996.

Hartley, R. and Zisserman, A. Multiple View Geometry in Computer Vision. Cambridge University Press, 2004. doi:10.1017/cbo9780511811685.

Joshi, S. M. Control of large flexible space structures, volume 131 of Lecture Notes in Control and Information Sciences. Springer, 1989. doi:10.1007/BFb0042076.

Kamel, A., Lange, F., and Hirzinger, G. New aspects of input shaping control to damp oscillations of a compliant force sensor. In 2008 IEEE International Conference on Robotics and Automation. pages 26292635, 2008. doi:10.1109/ROBOT.2008.4543609.

Kanestrom, R. and Egeland, O. Nonlinear active vibration damping. IEEE Transactions on Automatic Control, 1994. 39(9):1925-1928. doi:10.1109/9.317126.

Mun, J. I., Jo, T., Kim, T., and Pahk, H. J. Residual vibration reduction of white-light scanning interferometry by input shaping. Optics Express, 2015. 23(1):464. doi:10.1364/oe.23.000464.

Park, F. and Martin, B. Robot sensor calibration: solving $\mathrm{AX}=\mathrm{XB}$ on the euclidean group. IEEE Transactions on Robotics and Automation, 1994. 10(5):717721. doi:10.1109/70.326576.

Pérez, L., Rodríguez, Í., Rodríguez, N., Usamentiaga, R., and García, D. Robot guidance using machine vision techniques in industrial environments: A comparative review. Sensors, 2016. 16(3):335. doi:10.3390/s16030335. 
Preumont, A. Vibration Control of Active Structures. Springer Netherlands, 2011. doi:10.1007/978-94-0072033-6.

Rauscher, F., Nann, S., and Sawodny, O. Motion control of an overhead crane using a wireless hook mounted IMU. In 2018 Annual American Control Conference (ACC). IEEE, pages 5677-5682, 2018. doi:10.23919/acc.2018.8431170.

Robinett, R., R. Dohrmann, C., Richard Eisler, G., T. Feddema, J., G. Parker, G., Wilson, D., and Stokes, D. Flexible Robot Dynamics and Controls. Springer, 2002. doi:10.1007/978-1-4615-0539-6.

Savio, E., Chiffre, L. D., and Schmitt, R. Metrology of freeform shaped parts. CIRP Annals, 2007. 56(2):810-835. doi:10.1016/j.cirp.2007.10.008.

Schitter, G., Thurner, P. J., and Hansma, P. K. Design and input-shaping control of a novel scanner for high-speed atomic force microscopy. Mechatronics, 2008. 18(5-6):282-288. doi:10.1016/j.mechatronics.2008.02.007.

Schmitt, R., Peterek, M., Morse, E., Knapp, W., Galetto, M., Hrtig, F., Goch, G., Hughes, B., Forbes, A., and Estler, W. Advances in large-scale metrology - review and future trends. CIRP Annals, 2016. 65(2):643-665. doi:10.1016/j.cirp.2016.05.002.

Shiu, Y. and Ahmad, S. Calibration of wrist-mounted robotic sensors by solving homogeneous transform equations of the form $\mathrm{AX}=\mathrm{XB}$. IEEE Transactions on Robotics and Automation, 1989. 5(1):16-29. doi:10.1109/70.88014.

Siciliano, B., Sciavicco, L., Villani, L., and Oriolo, G. Robotics: Modelling, Planning and Control. Springer Publishing Company, Incorporated, 1st edition, 2008. doi:10.1007/978-1-84628-642-1.

Singer, N. C. Residual vibration reduction in computer controlled machines. Ph.D. thesis, MIT Artificial Intelligence Laboratory, Cambridge, MA, 1989.

Singer, N. C. and Seering, W. P. Preshaping Command Inputs to Reduce System Vibration. Journal of Dynamic Systems, Measurement, and Control, 1990. 112(1):76-82. doi:10.1115/1.2894142.
Singh, T. and Singhose, W. Tutorial on input shaping/time delay control of maneuvering flexible structures. In Proceedings of the 2002 American Control Conference (IEEE Cat. No.CH37301), volume 3. pages 1717-1731 vol.3, 2002. doi:10.1109/ACC.2002.1023813.

Singhose, W., Seering, W., and Singer, N. Residual vibration reduction using vector diagrams to generate shaped inputs. Journal of Mechanical Design, 1994. 116(2):654-659. doi:10.1115/1.2919428.

Singhose, W. E., Seering, W. P., and Singer, N. C. Input shaping for vibration reduction with specified insensitivity to modeling errors. Japan-USA Sym. on Flexible Automation, 1996. 1:307-13.

Song, Z., Guo, T., Fu, X., and Hu, X. Residual vibration control based on a global search method in a high-speed white light scanning interferometer. Applied Optics, 2018. 57(13):3415. doi:10.1364/ao.57.003415.

Tsai, R. and Lenz, R. A new technique for fully autonomous and efficient 3D robotics hand/eye calibration. IEEE Transactions on Robotics and Automation, 1989. 5(3):345-358. doi:10.1109/70.34770.

Umeyama, S. Least-squares estimation of transformation parameters between two point patterns. IEEE Transactions on Pattern Analysis and Machine Intelligence, 1991. 13(4):376-380. doi:10.1109/34.88573.

Vaughan, J., Yano, A., and Singhose, W. Comparison of robust input shapers. Journal of Sound and Vibration, 2008. 315:797-815. doi:10.1016/j.jsv.2008.02.032.

Yu Zhao, Chen, W., Te Tang, and Tomizuka, M. Zero time delay input shaping for smooth settling of industrial robots. In 2016 IEEE International Conference on Automation Science and Engineering (CASE). pages 620-625, 2016. doi:10.1109/COASE.2016.7743459.

Zhao, Y. and Tomizuka, M. Modified Zero Time Delay Input Shaping for Industrial Robot With Flexibility. American Society of Mechanical Engineers Digital Collection, 2017. doi:10.1115/DSCC2017-5219. 